A. Pramesh Rao, G. Swarup and Gopal-Krishna, eds.

\title{
Active Galaxies and Candidate Remnants in the Core of the Shapley Concentration
}

T. Venturi

Istituto di Radioastronomia, CNR, Via Gobetti 101, I-40129 Bologna

S. Bardelli

Observatorio Astronomico, Via Ranzani 1, I-40127 Bologna

D. Dallacasa

Dipartimento di Astronomia, Università di Bologna, Via Ranzani 1, I-40127 Bologna

R.W. Hunstead

School of Physics, University of Sydney, NSW 2006 Australia

R. Morganti

NFRA, Postbus 2, 7990 AA, Dwingeloo, The Netherland

T. Tzioumis

ATNF-CSIRO, Epping, NSW 2142, Australia

\begin{abstract}
We present preliminary results of a multifrequency and multiresolution study carried out with the Australia Telescope Compact Array for nine of the ten extended radio galaxies located in the merging cluster complexes A3558 and A3528, at the centre of the Shapley Concentration. We found that 5 out of the 9 extended radio galaxies are active radio galaxies, i.e. they have a clear active radio nucleus coincident with the central region of the associated optical galaxy, radio jets and extended lobes; the remaining four lack an obvious radio nucleus, have a very diffuse and amorphous morphology and exhibit peculiar spectral properties. We call these radio sources as remnants and propose that they are (a) either radio galaxies where the nuclear activity has ceased; or (b) regions where pre-existing electrons have been reaccelerated as consequence of shocks due to cluster mergers.
\end{abstract}

\section{Introduction}

In order to study the effects of cluster mergers on the the radio properties of the Shapley galaxies, in the past few years we have been surveying the two major cluster complexes in the central region of the Shapley Concentration, i.e. the 
A3558 and the A3528 groups, with the Australia Telescope Compact Array at 22/13 cm (Venturi et al. 1997, MNRAS 285, 898; Venturi et al., 1999 in Diffuse Thermal and Relativistic Plasma in Galaxy Clusters, MPE Report n. 271, p. 27; Venturi et al., 2000, MNRAS, in press). In the same region spectral information is available for $\sim 2000$ Shapley galaxies (Bardelli et al. 1998, MNRAS 300, 598; Bardelli et al., in preparation), as well as ROSAT and ASCA X-ray data. This unprecedented amount of data allows us (a) to study the statistical properties of the radio emission, and (b) to perform a detailed study of the extended radio galaxies resolving their true position within the cluster and the local condition. Our study on the A3558 complex shows a deficit of radio galaxies in this region.

\section{Radio Observations and Results}

From our $22 \mathrm{~cm}$ survey we found a total of 37 Shapley radio galaxies, 28 located in the A3558 complex (Venturi et al., 2000, MNRAS, in press) and the remaining 9 in the A3528 complex (Venturi et al., in prep.) We observed and imaged nine of the 10 extended Shapley radio galaxies with the Australia Telescope Compact Array at $22 \mathrm{~cm}, 13 \mathrm{~cm}, 6 \mathrm{~cm}, 3.6 \mathrm{~cm}$. The resolution of our observations ranges from $\sim 10^{\prime \prime} \times 6^{\prime \prime}$ at $22 \mathrm{~cm}$ to $\sim 1.7^{\prime \prime} \times 0.7^{\prime \prime}$ at $3.6 \mathrm{~cm}$. The nine radio galaxies under study are: J1254-2904, J1254-2900 and J1254-2901a in A3528N; J1254-2913 and J1254-2916 in A3528S; J1322-3146 and J1324-3138 in A3556; J1333-3141 and J1335-3153 in A3562. The source J1324-3138 has already been studied in detail, and the results are given in Venturi et al. 1998, MNRAS 298, 1113.

Our image analysis and study of their radio spectrum in the range $\alpha_{22 \mathrm{~cm}}^{3.6 \mathrm{~cm}}$ indicate that these radio galaxies differ from one another and are possibly in different stages of life. In particular we can distinguish between:

- radio galaxies with a clear active nucleus, coincident with the nucleus of the optical counterpart, jets and extended tails. Among these galaxies we find J1254-2900 and J1254-2913 (A3528); J1322-3146 (A3556); J1333-3141 and J1335-3153 (A3562).

- radio galaxies with amorphous and diffuse morphology. They lack an obvious nucleus, and have an optical counterpart within the radio isophotes. Their radio spectrum also is peculiar. These are J1254-2901, J1254-2904, and J1254-2916, all located in A3528.

We suggest that these three radio galaxies are:

(a) either remnants of radio galaxies, whose nuclear activity has now switched off, as we concluded for $\mathrm{J} 1324-3138$ in A3556;

(b) or regions where pre-existing electrons have been reaccelerated as a consequence of shocks due to clusters mergers. We remind here that there is evidence that the two components of A3528, i.e. A3528N and A3528S, are in a premerging stage, and the gas temperature gradients suggest a shock front in the region where J1254-2904, the most intriguing source in our list, is located.

In both cases cluster merger is likely to play a dominant role, either suppressing the nuclear activity of existing AGNs or providing the largest fraction of the energy requested to reaccelerate pre-existing electrons.

A detailed study on all extended radio galaxies presented in this paper is in progress. 